\title{
Mercado ¿Libre? en Argentina. La relativa autonomía de los usuarios-proveedores frente a su propio trabajo
}

\section{Mercado ¿Libre? in Argentina. The relative autonomy of users- suppliers over their own work}

ENSAYO

\section{Agustina Godoy}

Universidad Nacional de La Matanza, Argentina. Contacto: godoyagustina@gmail.com

Recibido: agosto de 2020

Aceptado: octubre de 2020

\section{Resumen}

Este ensayo, que tiene lugar en un contexto de auge de las plataformas digitales de consumo y de crecimiento del comercio electrónico, se propone reflexionar acerca de cómo la forma de la tecnología relativiza la autonomía de los usuarios-proveedores de Mercado Libre frente a su propio trabajo y cómo se construyen sus relaciones y sociabilidad. Para ello, se adopta una posición crítica acerca de la relación entre tecnología y sociedad en torno a la función social de las plataformas, el uso de los datos y de algoritmos y el derecho a la desconexión digital. Se exponen los argumentos a través del análisis de dimensiones tales como valores sociales, dimensión política, imaginario social y arquetipo en la memoria colectiva, así como la reconstrucción de los vínculos entre usuario-proveedor y usuario-cliente y usuario-proveedor y plataforma. Esta reflexión cobra relevancia en un escenario de plataformización de la economía que ha centrado el interés de la producción académica en las relaciones de trabajo de plataformas de consumo a demanda y que escasamente se adentra en las de comercio electrónico.

Palabras clave: Mercado Libre; Tecnología; Sociabilidad; Trabajo

\begin{abstract}
This essay, which takes place in a context of the rise of digital consumer platforms and the growth of e-commerce, aims to reflect on how the form of technology relativizes the autonomy of Mercado Libre users- suppliers from their own work and how their relationships and sociability are built. For that purpose, a critical position is adopted about the relationship between technology and society around the social function of platforms, the use of data and algorithms and the right to digital disconnection. The arguments will be presented through the analysis of dimensions such as social values, political dimension, social imaginary and archetype in the collective memory, as well as the reconstruction of the links between user-
\end{abstract}


supplier and user-customer and user-supplier and platform. This reflection becomes relevant in a scenario of the platformization of the economy that has focused the interest of academic production on the working relationships of consumer platforms on demand and that barely enters those of electronic commerce.

Keywords: Mercado Libre; Technology; Sociality; Work.

\section{Introducción}

Existe una tendencia mundial hacia la plataformización de la economía y de la vida cotidiana como devenir capitalista. Analizar este fenómeno desde las Ciencias Sociales implica adoptar una actitud crítica y consciente en torno a los debates contemporáneos acerca de la tecnología y la sociedad, tales como la función social de las plataformas, el uso de los datos y de los algoritmos y el derecho a la desconexión digital, entre otros.

El auge de estas plataformas suplanta al imaginario de androides que se sublevan frente al hombre y puede considerase como una expresión de la humanidad aumentada de Sadin (2017). En ella, el desarrollo de la ciencia, la técnica y la tecnología, especialmente de las de consumo, avanzan invisiblemente sobre la mercantilización de las diferentes esferas de la vida, a través de la extracción de datos y la automatización de la organización civilizatoria. Una de las características de estas plataformas es que, por medio de las nuevas tecnologías, se genera un espacio de encuentro virtual entre oferta y demanda para el intercambio de bienes y servicios no producidos por aquellas. Entre ellas, se encuentran gigantes tecnológicos relacionados con el comercio electrónico como Amazon de origen estadounidense y Alibaba Group de China.

En Argentina, la empresa mediadora que genera el mayor espacio de interacción de comercio electrónico entre usuarios-proveedores y usuarios-clientes es Mercado Libre. Fundada en 1999, actualmente está valuada en USD 50.000 millones, transformándose en una de las cinco empresas nacionales unicornio. ${ }^{1}$ Se define a sí misma como "la compañía tecnológica de comercio electrónico líder de América Latina" cuyo propósito es "democratizar el comercio y el dinero para impactar en el desarrollo de la región". ${ }^{2} \mathrm{Si}$ atendemos estrictamente al contenido de esta tecnología de consumo, las publicaciones de Mercado Libre ofrecen productos y servicios de quienes tienen intenciones de vender de manera online. Ahora bien, Mercado Libre no sólo representa la reconversión de una tienda física de compra y venta a una plataforma online, sino que, conforme a ser un grupo económicamente concentrado y sin competidores en el comercio electrónico a gran escala en el país, tiende a imponer reglas

\footnotetext{
${ }^{1}$ Empresas cuyo valor de mercado que superan los USD 1.000 millones. En Argentina, además de Mercado Libre se encuentran Globant, OLX, Despegar y Auth0.

2 Ver en Ideas Mercado Libre (4 de noviembre de 2020).
} 
de comercialización sin márgenes de negociación con otros actores. Al mismo tiempo, su tecnología se basa en el big data, ${ }^{3}$ la inteligencia artificial ${ }^{4}$ y el machine learning ${ }^{5}$ donde priman los algoritmos de posicionamiento y de preferencias que estructuran la forma de ver el contenido. ${ }^{6}$ También representa, a través de sus portavoces, los estandartes del emprendimiento en Argentina.

Legalmente, se reconoce a la plataforma de Mercado Libre como intermediaria en una relación de consumo. Sin embargo, su comportamiento no es inocuo para quienes trabajan comercializando bienes y servicios a través de ella: establece las condiciones para el intercambio en términos de precios o comisiones, formas de pago, tiempos y modo de entrega, reputación digital, entre otros aspectos. ${ }^{7}$ A su vez, ofrece su motor de búsqueda y almacena datos personales, transaccionales y de preferencias. $Y$ principalmente, considera a los usuarios-proveedores como empresarios de sí mismos, otorgándoles libertad en la determinación del volumen y momento de la prestación de servicios, junto con la utilización de sus propios medios de producción (Ginès i Fabrellas, 2016).

Si bien, como dijimos, Mercado Libre se presenta como la "compañía tecnológica de comercio electrónico líder de América Latina”, este mensaje explícito debe ser puesto en consideración junto a una dimensión más implícita de análisis: la forma de la tecnología (Ringelheim, 2020) y las relaciones y sociabilidad que devienen de ella. En virtud de esto, el objetivo de este ensayo es evidenciar cómo la forma de la tecnología relativiza la autonomía de los usuarios-proveedores de Mercado Libre frente a su propio trabajo y cómo se construyen las relaciones y sociabilidad entre los usuarios y la plataforma.

\footnotetext{
${ }^{3}$ Es la combinación de algoritmos que buscan crear máquinas con capacidades similares a las del ser humano. Fuente: Definiciones de Big Data e Inteligencia Artificial de la I a la Z (18 de febrero de 2020).

${ }^{4}$ Hace posible que las máquinas aprendan de la experiencia y realicen tareas como hacen los humanos. Estos procesos incluyen el aprendizaje, el razonamiento y la autocorrección. Fuente: Definiciones de Big Data e Inteligencia Artificial de la I a la Z (18 de febrero de 2020).

${ }^{5}$ Es la combinación de disciplinas científicas para crear sistemas que aprendan sin necesidad de intervención humana. Es decir, que sean capaces de identificar patrones complejos en millones de datos, pero que al mismo tiempo sean capaces de afinar su desempeño de forma autónoma y progresiva. Fuente: Definiciones de Big Data e Inteligencia Artificial de la I a la Z (18 de febrero de 2020).

${ }^{6}$ Cabe destacar que Mercado Libre, a diferencia de Amazon, no posee servicios en la nube como Amazon Web Services (AWS) y contrata este servicio de infraestructura tecnológica que le permite, entre otras cuestiones, operar online y enviar sus datos crudos de consumo, geolocalización y capacidad de pago y, a cambio, demanda inteligencia artificial.

${ }^{7}$ Mercado Libre aún no ha incursionado en la venta de productos de su propia marca a través de su plataforma, tal como lo ha hecho Amazon que comercializa sus productos y es observado en varios países por prácticas desleales. Sin embargo, Mercado Libre sí cuenta con su propia tienda de Mercado Libre Electrónica donde comercializa productos tecnológicos de diferentes marcas y compite con otras tiendas o proveedores.
} 


\section{La forma implícita de una tecnología de consumo: el caso de Mercado Libre}

Para analizar la forma implícita de Mercado Libre como tecnología de consumo, en primer lugar, se toma en cuenta su relación con diferentes aspectos del entramado social, propuestos por Ringelheim (2020), tales como, los valores sociales que pregona, su dimensión política, el imaginario social que se construye y el arquetipo que tiene lugar en la memoria colectiva. Esta forma influye en la participación de los usuarios, por medio de lo que de Van Dijck (2016) denomina como "hábito" de quienes programan la plataforma y tienen injerencia, por ejemplo, en los algoritmos que orientan al consumidor en sus gustos y preferencias.

En cuanto a los valores, podemos pensar que Mercado Libre se arraiga en los principios de la New Economy de los años 90, donde el sujeto trabajador se convierte en un empresario de sí mismo tal como señala Berardi (2003). Lo hace tras "una promesa de felicidad individual, de éxito asegurado, de ampliación de los horizontes de experiencia y de conocimiento" (p. 10). Esta forma de empresa es el principio que vertebra las relaciones sociales en el neoliberalismo. En el modelo de Mercado Libre, el empresario es el proveedor de bienes o servicios que no tiene subordinación en términos de jornada y horarios, más que su propia demanda personal. Los trabajadores se convierten en emprendedores de sus micro-empresas, individualizados, sin acceso a derechos laborales ni sindicales. $Y$ en el marco de los debates actuales, ${ }^{8}$ tampoco poseen derechos a ningún tipo de desconexión digital que les permita mantener un balance entre la vida personal y el trabajo, sin que ello modifique su performance en la plataforma.

Cabe destacar que la autonomía empresarial de los usuarios-proveedores es relativa. Tal como se detallará con más precisión en el siguiente apartado, la plataforma es la que determina indirectamente el precio del producto a través de las comisiones por venta, formas y tiempos de cobro, modos y tiempo de entrega; en tanto que también sugiere formas y tiempos de respuesta. Estas condiciones se conjugan con la creación de una reputación digital, las tiendas oficiales y el pago de publicidad; en tanto, el algoritmo secreto hace su trabajo: determina el orden y la disposición de las publicaciones. Tal como señala Van Dijkc (2016), los "algoritmos suelen ser secretos comerciales de sus propietarios, similares a las patentes $\mathrm{u}$ otros tipos de propiedad intelectual" y con su programación "infiltran una (trans)acción social por medio del análisis de datos computacionales, a partir del cual el resultado se traduce en una táctica sociocomercial" (p. 35).

\footnotetext{
${ }^{8}$ Con la pandemia del covid-19 se han intensificado los debates acerca de las nuevas formas de empleo y las regulaciones que se requieren para ejercer el derecho a conectarse y desconectarse de las aplicaciones. Por un lado, el derecho a la conectividad garantiza el ejercicio otros derechos como la educación y el trabajo y, por otro lado, el derecho a la desconexión reafirma la intimidad personal del trabajador fuera de la jornada regular de trabajo.
} 
En tanto que el comercio electrónico $\mathrm{crece}^{9}$ y se produce un mayor uso de la plataforma, esos datos transaccionales y de geolocalización se convierten en la materia prima de la economía digital y de la maximización de los beneficios económicos que pueden obtener las empresas. En otras palabras, el uso que se hace de estos datos comprende a la dimensión política de la plataformización. En ella, Mercado Libre no es sólo una empresa privada de tecnología o de comercio electrónico, ${ }^{10}$ sino que por su concentración económica es un actor social que disputa el poder en búsqueda de la racionalización productiva. A su vez, frente a la escasez de competidores impone reglas de comercialización y direcciona las preferencias de consumo algorítmicamente, lo que diluye su imperativo de democratizar el comercio. De acuerdo con Zuazo (2018), no existe una mayor democratización por el hecho de que la tecnología nos medie. La autora sostiene que para que realmente los beneficios sociales, originados por los avances tecnológicos, se den de forma extendida se debe politizar la tecnología y el Estado debe intervenir. En la misma línea, pero más crítica aún, Srnicek (2018) también se enfoca en la función social de las plataformas y sostiene que hay que regular a las grandes empresas tecnológicas o convertirlas en servicios públicos, en tanto que tienden naturalmente hacia la monopolización y puedan convertirse en el comercio electrónico donde se compra y vende todo, como lo es Amazon a nivel mundial o como podría ser Mercado Libre, a nivel regional.

En relación con lo anterior, una incipiente respuesta estatal del actual gobierno del presidente Alberto Fernández -que se muestra más como una estrategia tradicional de romper el monopolio antes que reconocer a la plataforma como servicio público- fue el reciente lanzamiento de Correo Compras, ${ }^{11}$ una tienda virtual que conecta directamente a productores con consumidores de todo el país. Si bien, a diferencia de Mercado Libre, en Correo Compras sólo pueden comercializar empresas, existe un beneficio de mayor alcance social implícito: el tratamiento que se le pudieran hacer a los datos recabados para diseñar políticas públicas.

\footnotetext{
${ }^{9}$ También como consecuencia de las medidas de distanciamiento social, a raíz de la pandemia del covid-19, el e-commerce creció exponencialmente en todo el mundo. En Argentina, de acuerdo con el balance del tercer trimestre de Mercado Libre, duplicó su cantidad de usuarios y su facturación creció $85 \%$ en dólares. Fuente: "Mercado Libre, imparable en la pandemia: casi duplicó usuarios y su facturación creció 85 por ciento en dólares" (2020, 04 de noviembre).

${ }^{10}$ En 2017, existió una discusión en torno a si Mercado Libre era una empresa de software o de comercio electrónico. La Administración Federal de Ingresos Públicos (AFIP) cuestionó su incorporación en el régimen de Ley № 27.506 de Economía del Conocimiento. En la actualidad, si bien existe un consenso en reconocer a Mercado Libre como una empresa de tecnología, la nueva Ley $N^{\circ} 27.570$ de Economía del Conocimiento, promulgada el 25/10/2020, modifica ciertos puntos para favorecer el estímulo hacia las pymes tecnológicas. Por ejemplo, que la reducción en el pago del impuesto a las ganancias que antes comprendía el $60 \%$ para todos los beneficiarios, ahora tenga lugar de forma segmentada: el $60 \%$ de descuento para pymes y micropymes, $40 \%$ para las medianas y $20 \%$ para las más grandes.

${ }^{11}$ En 24 horas de su puesta en marcha, la plataforma realizó transacciones por 7.410 .000 pesos, hubo 1.014 encargos y 2.000 empresas y productores locales que pidieron sumarse. Fuente: "Correo Compras, un Mercado Libre sin intermediarios" (Russo, 2020, 17 de octubre).
} 
En otro orden y siguiendo con el análisis de la forma implícita, al pensar en el imaginario social como una "creación incesante y esencialmente indeterminada de figuras/formas/imágenes" (Castoriadis, 2013, p. 12), Mercado Libre se convierte en uno de los imaginarios que legitima socialmente al capitalismo neoliberal a través de la libertad y, especialmente, del libre mercado promocionado por los avances tecnológicos. Un libre comercio, en este caso electrónico, que conlleva un mayor alcance global y que lo convierte en una tienda sin limitación geográfica, capaz de concretar ventas en todo momento. Es expresión de velocidad, deseo y éxito individual del emprendedor digital.

Ahora bien, este espíritu emprendedor al que hacemos referencia se reproduce a través de portavoces capaces de dirigir la conducta de los sujetos: el director y otros ejecutivos de la firma, los jóvenes emprendedores que cuentan sus historias de éxito y también economistas, periodistas, políticos, entre otros. ${ }^{12}$ Sus mensajes circulan a través del semiocapitalismo (Berardi, 2003) que promueve la acumulación de bienes inmateriales que actúan sobre la mente colectiva. Este semiocapitalismo tiene origen en la inmaterialización de la economía capitalista por medio de la financiarización y en la virtualidad semiótica que se despoja de los referentes. De acuerdo con Forster (2018), los "lenguajes de la virtualidad y las tecnologías digitales han avanzado de manera sistemática sobre las estructuras más profundas de la subjetividad" (p. 506). De esta forma, los discursos acerca del emprendedor entusiasta se manifiestan como discursos de la verdad que impactan directamente en el proceso de subjetivación de los usuarios-proveedores y también de los usuarios-clientes de Mercado Libre.

Por último, mencionamos que las plataformas virtuales como Mercado Libre reinauguran aquella idea moderna y humanista del progreso indefinido de una sociedad $\sin$ riesgos. ${ }^{13}$ Alientan a un nuevo optimismo tecnológico que se desentiende de las crisis económicas y medioambientales. Para Sadin (2018) existe un espíritu de Silicon Valley que emergió como contracultura en los años 60 en California y que hoy es una ideología dominante que se expande mundialmente. Su objetivo es colonizar los datos para monetizar todas las esferas de la vida y organizar algorítmicamente a la sociedad. En este punto las empresas start-up cumplen un papel determinante como arquetipo en el inconsciente colectivo. Regidas por este espíritu siliconiano de emprendimiento digital, construyen el relato acerca de que las innovaciones para cambiar el mundo nacen en un garage. $Y$ así comienza la historia de Mercado Libre, creada en 1999 en un garage del barrio de Saavedra, Ciudad Autónoma de Buenos Aires.

\footnotetext{
12 Pueden leerse casos de éxito en la sección de Ideas Mercado Libre. "Mercado Libre y Endeavor premiaron a los mejores emprendedores de Latinoamérica" (18 de octubre de 2020)

${ }^{13}$ De acuerdo con Beck (1986), sociedad del riesgo es la condición humana del siglo XXI.
} 


\section{El devenir de la forma: relaciones y sociabilidad entre usuario-proveedor y usuario- cliente y usuario-proveedor y plataforma}

En este apartado analizaremos el devenir de la forma de la tecnología, a partir de la interacción que hace el sujeto con la plataforma y con otros sujetos, en términos de relacionamiento y sociabilidad. Para ello, examinaremos las relaciones entre usuarioproveedor y usuario-cliente y usuario-proveedor y plataforma.

En principio, recuperamos la frase de McLuhan (1982) para quien el medio es el mensaje. Esta afirmación requiere estudiar las implicancias que la forma de un medio plasma en el mensaje. Cualquier intención de dar cuenta acerca del fenómeno de Mercado Libre, tendrá sentido sólo si el medio es analizado en función del entramado social, cultural y tecnológico y si comprendemos su capacidad de modificar la conducta humana a través de su imbricación con el mensaje.

Si bien los medios en auge han cambiado desde la época en la que escribió McLuhan, la naturaleza de los nuevos medios nos remite a la misma idea que planteaba el autor: los medios tecnológicos se convierten en una extensión de nuestros sentidos como, por ejemplo, la vista o el tacto. Y si bien esto no implica necesariamente un aumento de nuestras habilidades físicas, lo que sí se expande es nuestra habilidad de percibir un mundo de una forma que sería imposible sin el uso de estos medios.

De esta manera, por medio de una computadora o celular, un consumidor puede percibir en Mercado Libre una oferta y variedad de productos mucho más amplia de la que podría encontrar movilizándose físicamente a diferentes comercios e incluso navegando por diferentes tiendas online. Ahora bien, esta percepción ampliada, tal como se verá más adelante, estará condicionada por los motores de búsqueda de Mercado Libre que muestran de forma organizada los resultados en una página en menos de un segundo y cuyo orden responden a algoritmos de posicionamiento.

Si para McLuhan, el medio es el mensaje, para Virilo (1996) el medio es la velocidad y las plataformas virtuales nos ofrecen la posibilidad de acceder a los objetos de consumo superando la barrera del aquí en favor del ahora. El e-commerce hace que el sujeto ya no necesite salir de su casa para comprar; esto transforma la relación entre la persona que vende y quien compra. Ambos mantienen sus roles, pero, además, se convierten en usuarios de la plataforma: usuario-proveedor y usuario-cliente.

Al mismo tiempo, se genera una pérdida del trayecto en relación con el objeto, donde también se pierde la relación con los otros cuerpos y con lo sensorial. En otras palabras, lo que se pierde, en términos de Berardi (2017), es la relación conjuntiva que intercambia signos y contexto, en este caso, entre el vendedor y el comprador. El autor sostiene que "en la virtualización la presencia del cuerpo del otro se vuelve superflua, cuando no incómoda y 
molesta (...) el otro debe aparecer como información, como virtualidad y, por tanto, debe ser elaborado con rapidez y evacuado en su materialidad" (p. 184).

De hecho, las partes sólo pueden intercambiar datos de contacto una vez concretada la venta en la plataforma. El vendedor puede observar "nombre" de usuario (que pocas veces remite a un nombre personal) únicamente al momento de responder una pregunta y no puede contestar la misma pregunta más de una vez. Se suprime la capacidad argumental o, al menos, queda limitada a una serie de caracteres - sin kinestesia, sin voz y $\sin$ tonalidad condicionados, a su vez, por la velocidad de respuesta que tácitamente se exige para mantener una buena reputación digital. Lo que existe entre ambas partes es una relación puramente sintáctica en el intercambio de preguntas y respuestas, despojándose de cualquier componente situacional. La argumentación racional humanista, que debería ser potestad del usuario-proveedor "empresario de sí" cede ante la distancia de los cuerpos mediados por la pantalla de la era posthumana.

En cuanto a la relación entre usuario-proveedor y plataforma, ésta última impone las reglas de comercialización y tiene nulos márgenes de negociación con otros actores. Esto último se debe, principalmente, a la ausencia de presiones que pudiera recibir de otros competidores y de las demandas de los usuarios que, por necesidades de trabajar, no pueden prescindir de la plataforma. De los términos y condiciones de Mercado Libre, se desprende que si bien en principio el usuario-proveedor de la plataforma es quien define el valor de su servicio, los proveedores estándar que no disponen de una tienda oficial deben pagar comisiones ${ }^{14}$ que van desde el $13 \%$ y el $28 \%$ si incluye hasta 6 pagos con tarjeta de crédito (con un adicional de $\$ 15$ por unidad en ventas menores de \$2.500). Es cierto que Mercado Libre no cobra por publicar, sino por las ventas realizadas de acuerdo con la exposición elegida previamente. En este sentido, se paga un canon por el costo de venta del producto una vez concretada la operación.

Aquellos usuarios-proveedores que eligen la exposición gratuita tendrán alcance nulo en el posicionamiento del motor de búsqueda y quedarán imperceptibles para los consumidores. Aun así, si la publicación fuera con exposición gratuita y el vendedor superase las barreras del posicionamiento, sólo podrá publicar de esta forma hasta alcanzar las cinco ventas en el último año y luego deberá elegir una membresía paga.

Para las tiendas oficiales, que generalmente involucra a las grandes marcas, los costos por venta del producto son diferentes: $10 \%$ y $26 \%$ si incluye hasta 6 pagos con tarjeta de crédito (con un adicional de $\$ 15$ por unidad en ventas menores de $\$ 2.500$ ). Es interesante preguntarse por qué las comisiones son superiores para los usuarios estándar -los más pequeños- cuando el objetivo de Mercado Libre, al menos como slogan empresarial, es la democratización del comercio.

${ }^{14}$ Ver en Ayuda Mercado Libre: Costos de vender un producto. 
A su vez, Mercado Libre ofrece anuncios publicitarios pagos que permiten destacar las publicaciones. Incluso, mientras un usuario esté navegando en la publicación de otro vendedor podrá encontrar en esa misma pantalla dichas publicaciones destacadas.

En relación con los pagos, ${ }^{15}$ Mercado Libre retiene el dinero entre cinco y nueve días de acuerdo con la reputación del vendedor y el modo de envío. Además, premia con una reputación de "Mercado Líder" ${ }^{16}$ dividida en tres niveles que ofrece una serie de ventajas, entre ellas, mayor prioridad y exposición en las búsquedas. Para obtener esta distinción se deben cumplir varios requisitos, sostenidos al menos por tres meses y en forma simultánea. Ente las condiciones solicitadas se combinan mínimos para la cantidad de ventas y la facturación total de las ventas concretadas (en las que se excluyen aquellas conseguidas con exposición gratuita), reputación (color del termómetro verde), ínfimos porcentajes de reclamos, cancelaciones y demoras en los envíos. De esta forma, un usuario-proveedor que vendió 50 productos en los últimos tres meses y que cumple con los mínimos referidos al color de la reputación, reclamos, cancelaciones y demoras en los envíos, requiere, además, que su producto valga al menos $\$ 2.000$ por unidad para alcanzar los $\$ 100.000$ que requiere la primera distinción de "Mercado Líder".

Este juego de membresías, reputaciones y distinciones digitales tiene lugar, a través de un algoritmo secreto, para determinar el posicionamiento de las publicaciones en el motor de búsqueda de Mercado Libre. Si bien existen indicaciones públicas de cómo mejorar el posicionamiento, el efecto de bola de nieve ${ }^{17}$ favorece a las grandes tiendas y las llevará a tener siempre una mejor ubicación dentro de las ofertas. Basta con realizar la búsqueda de un producto y observar cuáles son los primeros resultados que percibimos.

También es importante destacar que, a diferencia de otras plataformas a demanda como Uber, Rappi, Glovo, entre otras, Mercado Libre no se enfrenta a discusiones acerca de la existencia o no de relaciones de trabajo y la calidad de ésta entre usuarios y plataforma. ${ }^{18}$ Esto se debe a que opera en un sector en el que inicialmente predominaba el trabajo independiente como el de los pequeños comerciantes (Madariaga, Molina y Ernst, 2019), aunque luego se haya extendido también a servicios profesionales. Tampoco está definido su rol en las relaciones de consumo y, aunque existe jurisprudencia acerca de su responsabilidad, no es concluyente. ${ }^{19}$

Aun así, puede percibirse cierta subordinación por parte de los usuarios-proveedores o, al menos, una relativa autonomía frente a su trabajo dada la imposibilidad de una desconexión

\footnotetext{
${ }^{15}$ Ver en Ayuda Mercado Libre: Cómo cobrar con cada medio de pago Ayuda Mercado Libre.

${ }^{16}$ Ver en Ayuda Mercado Libre: Qué es y cómo funciona la reputación como vendedor en Ayuda.

${ }^{17}$ Mientras más usuarios visualicen y compren en la tienda, atraerá a más usuarios y tendrá un mejor posicionamiento en el motor de búsqueda.

${ }^{18} \mathrm{Si}$ bien no forma parte de este análisis, uno de los servicios de entrega de Mercado Libre es Mercado Flex y allí los conductores pueden registrarse para realizar entregas, de modo similar a las otras apps que sí están en discusión.

${ }^{19}$ La jurisprudencia argentina es explicada por Domínguez Ginestar (2018).
} 
digital, sin que ello modifique su performance dentro de la plataforma. En definitiva, al analizar la dimensión implícita de la forma con la que opera la plataforma y las relaciones y sociabilidad que devienen de ella, se advierte que los usuarios-proveedores, lejos de ser "empresarios de si", quedan subordinados a la lógica de la plataforma. Lo que se observa, entonces, es que la idea original de Mercado Libre como "compañía tecnológica de comercio electrónico para democratizar el comercio" actúa como un mensaje disociado de la realidad de los usuariosproveedores.

\section{Conclusiones}

De acuerdo con lo expuesto, podemos concluir, en primer lugar, que la forma de la tecnología de consumo Mercado Libre, construye su modelo de negocios el espíritu siliconiano de emprendimiento digital, divulgado en los casos de éxito, que convierte a los comerciantes en "empresarios de sí". A su vez, la sociabilidad que deviene del uso la plataforma plantea relaciones virtuales y enigmáticas con el otro, alejando a las partes de un intercambio argumental y sensitivo, característico de la acción comercial clásica de quien vende y compra.

En segundo lugar, las reglas de comercialización de la plataforma están escasa o nulamente sujetas a otros competidores o demandas que los usuarios pudieran realizar para generar un cambio. Estas reglas de comercialización afectan, radicalmente, a los pequeños usuarios-proveedores que, además, dependen de comercializar en la plataforma para obtener una fuente de ingresos. Principalmente, si asumimos que las grandes tiendas tienen ciertas ventajas directas por sobre los pequeños usuarios-proveedores, tales como infraestructura, cantidad de empleados disponibles para rotar su disponibilidad online, recursos económicos para solventar publicidad, reconocimiento de la marca, entre otros. Y otras más indirectas y no tan a las sombras de las primeras: mayores posibilidades de alcanzar distinciones en la plataforma, así como un posicionamiento prácticamente exclusivo en el motor de búsqueda de Mercado Libre. Como hemos visto, esto lo resuelve un algoritmo secreto que organiza visualmente los productos en un determinado orden para que puedan ser percibidos por más clientes y así aumentar sus posibilidades de vender. $Y$ aunque el algoritmo es secreto, su finalidad es maximizar los beneficios económicos. De aquí que la intervención del Estado resulte fundamental para determinar la función social de las plataformas y tomar acciones en virtud de ello.

Finalmente, podemos concluir que las reglas de comercialización de Mercado Libre influyen, principalmente, en la autonomía de los pequeños usuarios-proveedores frente a su propia organización del trabajo: existe un direccionamiento sobre los tiempos para responder consultas, sobre los tiempos de despachos y envíos, sobre los tiempos para la liberación de un pago. A su vez, en la medida en que estos usuarios-proveedores consiguen cruzar el umbral del posicionamiento y ganar una mínima reputación digital, también van creando una mayor 
dependencia económica de sus ventas. Esto lleva a pensar que en tanto el trabajo siga ligado a la subsistencia, el costo que deben afrontar por tener más ventas no es sólo económico en términos del canon que cobra Mercado Libre, ni tampoco de prestigio como ser denominados "emprendedores de sí". Es un costo de pérdida de libertad, de sujeción virtual donde no hay posibilidades a la desconexión digital. Si pensamos en Foucault (1989), el usuario-proveedor se convierte en un sujeto sujetado por una fuerza que excede a su propia conciencia racional, en este caso, la forma de la tecnología de plataformas que constituye su propia subjetividad. Una sujeción al estilo de la película Tiempos Modernos de Chaplin, donde la famosa cinta de montaje, esta vez, comienza por el tiempo de respuesta detrás de una pantalla.

\section{Referencias bibliográficas}

Ayuda Mercado Libre. Mercado Libre. Cómo cobrar con cada medio de pago. Recuperado https://bit.ly/2IOKjvx

Ayuda Mercado Libre. Mercado Libre. Costos de vender un producto. Recuperado de: https://bit.ly/32QVL13

Ayuda Mercado Libre. Mercado Libre. Qué es y cómo funciona la reputación como vendedor. Recuperado de: https://bit.ly/3pDU4hl

Beck, U. (1998). La sociedad del riesgo. Hacia una nueva modernidad (2da ed). Barcelona, España: Paidós.

Berardi, F. (2003). Globalismo inhumano, horizonte posthumano. En La fábrica de la infelicidad. Nuevas formas de trabajo y movimiento global. Madrid: Traficantes de sueños.

Berardi, F. (2017). Fenomenología del fin. Sensibilidad y mutación conectiva. Buenos Aires: Caja Negra.

Castoriadis, C. (2013). La institución imaginaria de la sociedad. Barcelona: Tusquets.

Definiciones de Big Data e Inteligencia Artificial de la I a la Z (18 de febrero de 2020). Portal B12 Tech4 Busines. Recuperado de: https://bit.ly/38h1cK9

Domínguez Ginestar, M. (2018). Contratación electrónica y las plataformas de comercio electrónico", XVIII Simposio Argentino de Informática y Derecho (SID) - JAIIO 47 (CABA, 2018). Forster, R. (2018). El triunfo de la abstracción: semiocapitalismo y dominación neoliberal. En Revista Derechos en Acción, 3(8), pp. 503-515. DOI: 10.24215/25251678e197

Foucault, M. (1989). Vigilar y castigar. Buenos Aires: Siglo XXI.

Ginès i Fabrellas, A. (2016). Externalización productiva y elusión de compromisos laborales. La necesidad de revisar la normativa europea en materia de subcontratación y sus consecuencias laborales. En Revista Internacional y Comparada de Relaciones Laborales y Derecho del Empleo, 4(1).

Ideas Mercado Libre (4 de noviembre de 2020). Mercado Libre. Recuperado de: https://bit.ly/38MUjRb 
Ideas Mercado Libre (18 de octubre de 2018). Mercado Libre. Recuperado de: https://bit.ly/2/4j14F

Madariaga, J., Buenadicha, C., Molina, E. y Ernst, C. (2019). Economía de plataformas y empleo ¿Cómo es trabajar para una app en Argentina?, CIPPEC-BID - OIT. Buenos Aires.

McLuhan, M. (1982). Introducción, El medio es el mensaje, "El enamorado de los dispositivos", en La comprensión de los medios como las extensiones del hombre. México: Diana.

Mercado Libre, imparable en la pandemia: casi duplicó usuarios y su facturación creció 85 por ciento en dólares (2020, 04 de noviembre). Infobae. Recuperado de https://bit.ly/38nEErb

Virilio, P. (1996). Del superhombre al hombre sobreexcitado, en El arte del motor. Buenos Aires: Manantial.

Ringelheim, J. P. (2020). Clase 4: Amputaciones, prótesis y ciborgs. En Problemas Contemporáneos de la Tecnología y la Sociedad, Maestría en Ciencias Sociales y Humanidades, Universidad Nacional de Quilmes. Inédito.

Russo, N. (2020, 17 de octubre). Correo Compras, un Mercado Libre sin intermediarios. Página 12. Recuperado de: https://bit.ly/32fxuRO

Sadin, E. (2017). Del sujeto humanista al individuo algorítmicamente asistido, en La humanidad aumentada. La administración digital del mundo. Buenos Aires: Caja Negra Editora.

Sadin, E. (2018). La silicolonización del mundo. La irresistible expansión del liberalismo digital (Trad. de M. Martínez). Buenos Aires: Caja Negra Editora.

Srnicek, N. (2016). Capitalismo de plataformas. Buenos Aires: Caja Negra Editora.

Zuazo, N. (2018). Los dueños de internet: Cómo nos dominan los gigantes de la tecnología y qué hacer para cambiarlo. Buenos Aires: Debate. 